\title{
MÉTAMATÉRIAUX \\ pour la protection sismique
}

\author{
Sébastien GUENNEAU ${ }^{1}$, Stefan ENOCH ${ }^{1}$, Andrea COLOMBI ${ }^{2}$, Philippe ROUX ${ }^{3}$, Stéphane BRULE ${ }^{4}$ \\ ${ }^{1}$ Aix Marseille Univ, CNRS, Centrale Marseille, Institut Fresnel, Marseille, France $-{ }^{2}$ Dept. of Mathematics, Imperial \\ College London, UK $-{ }^{3}$ ISTerre, CNRS, Univ. Grenoble Alpes, Grenoble, France $-{ }^{4}$ Ménard, Nozay, France \\ sebastien.guenneau@fresnel.fr
}

Les physiciens des ondes élaborent des modèles théoriques et numériques pour un contrôle accru de la lumière dans des matériaux structurés à l'échelle nanométrique et s'en inspirent pour contrôler les ondes mécaniques de Rayleigh dans des sols structurés à l'échelle métrique, avec des trous dans un sol ou des arbres en surface savamment agencés. Un transfert de paradigme s'opère entre les métamatériaux électromagnétiques pour la photonique et les métamatériaux sismiques pour le génie civil.

\section{Métamatériaux pour les ondes électromagnétiques}

Les opticiens savent aujourd'hui conférer à un matériau composite l'anisotropie nécessaire pour contrôler presqu'à loisir la trajectoire et la vitesse de la lumière se propageant en son sein. À titre d'exemple, les fibres optiques ont accompagné la révolution de l'Internet ces vingt dernières années, et ont valu un prix Nobel de physique à l'ingénieur américano-britannique Charles Kao en 2009. Dans les années à venir, la propagation quasiment sans dispersion ni pertes de la lumière dans le cœur creux d'une nouvelle génération de fibres dites micro-structurées ouvrira de nombreuses perspectives telles que de nouvelles sources. Mais il y a plus étonnant encore : le phénomène de réfraction négative, prédit par le physicien moscovite Victor Veselago en 1968, permet de focaliser la lumière à travers une lentille plate avec une résolution sub-longueur d'onde. Ce résultat surprenant a été établi en 2000 par le physicien britannique Sir John Pendry, à qui l'on doit également le concept des capes d'invisibilité dans lesquelles les ondes suivent des trajectoires courbes, ce que l'on peut rapprocher des géodésiques de la lumière au voisinage des corps massifs célestes dans la théorie de la relativité générale d'Einstein. La question qui s'est posée à nous il y a quelques années est la possible transcription de la physique des métamatériaux électromagnétiques aux ondes sismiques. Cette possible analogie, qui n'est pas nécessairement triviale, s'appuie sur des modèles dits de masses et ressorts qui sous-tendent la physique des milieux localement résonants.

Les fibres micro-structurées ont été introduites par le physicien britannique Philip Russell dans les années 90, et exploitent la bande interdite d'un cristal photonique entourant le cœur [1].

\section{Physique transformationnelle et analogies pour les différents types d'ondes}

\section{Invisibilité versus protection}

Les mêmes techniques de transformation d'espace peuvent être utilisées pour rechercher des structurations théoriques de la matière à vocation de " protection ». Des phénomènes aussi surprenants que rendre un objet invisible sont accessibles à ces techniques de transformation d'espace. La figure 1 avec une cape d'invisibilité de $20 \mathrm{~cm}$ de diamètre

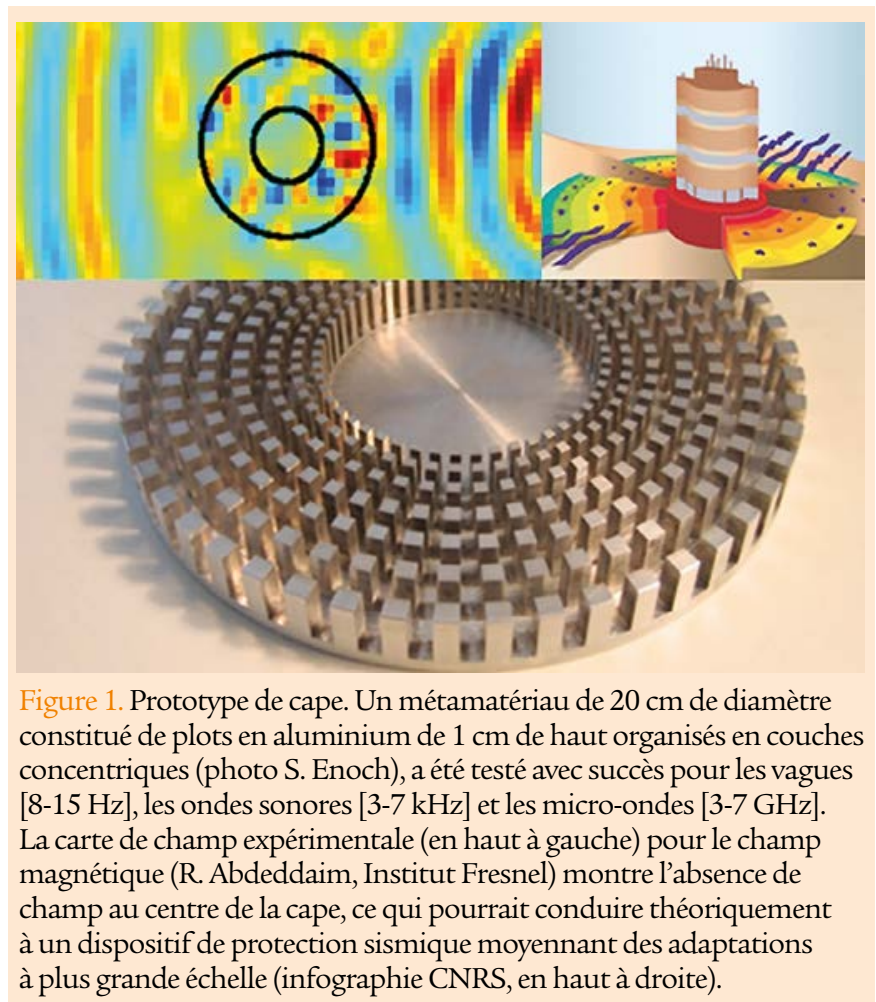




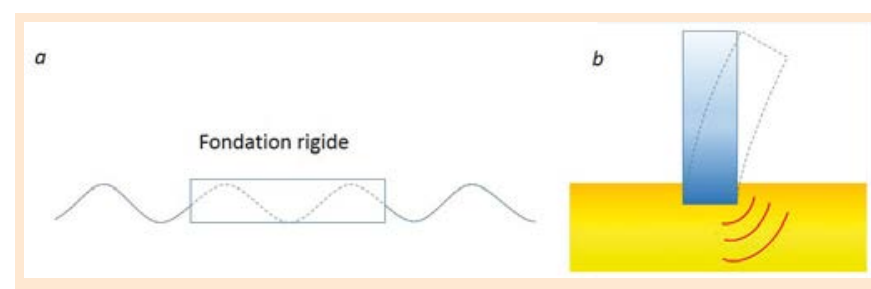

Figure 2. Interaction cinématique d'une fondation rigide avec le mouvement en champ libre du sol (a). Interaction inertielle avec génération de sources secondaires à la base des fondations (b).

en aluminium réalisée en 2008, illustre cette recherche dans les cas de l'hydrodynamique, de l'acoustique et de l'électromagnétisme.

De façon plus générale, la matière structurée est constituée d'un matériau anisotrope très hétérogène mettant en jeu des phénomènes d'interférence. Au niveau macroscopique, tout se passe comme si ce complexe était constitué d'un matériau homogène aux propriétés exotiques. Dans certaines configurations, l'indice de réfraction (dit effectif) de ce qu'il convient d'appeler un métamatériau pourra prendre des valeurs inférieures à 1 (voire négatives) quand la fréquence est en résonance avec des briques élémentaires telles que des boucles métalliques (ou anneaux) fendus introduits par Pendry en 1999 pour du magnétisme artificiel [2] qui constituent l'ossature du dispositif. En pratique, l'absorption et l'impossibilité de travailler à une seule longueur d'onde évitent tout paradoxe ou bizarrerie : l'effet « magique » du métamatériau ne se produit qu'autour d'une fréquence et les métaux constituant les résonateurs sont fortement dissipatifs aux longueurs d'ondes optiques. Il y aura bien un retard (faiblement) mesurable induit par le déphasage entre le front d'onde qui contourne le dispositif et celui qui se propage en espace libre.

La transformation d'espace a pour conséquence la création d'un volume central " protégé » (ou zone d'exclusion) du rayonnement au sein du dispositif. Toutefois, ceci n'est exact qu'à une fréquence donnée dépendant de l'épaisseur du dispositif et de la taille des résonateurs qui, dans le cas des ondes électromagnétiques, sont des boucles métalliques fendues. La condition d'existence du phénomène est l'excitation des résonances internes de ces éléments et un dispositif structuré d'épaisseur suffisante.
Un objet est « invisible » s'il ne modifie pas, ou peu, le champ d'onde dans lequel il est placé. Soit l'objet lui-même détient ces propriétés de reconstruction du champ d'onde comme s'il n'était pas présent dans l'espace considéré (il est alors question de " transparence »), soit il est entouré d'un dispositif (une "cape ») offrant ce même avantage. L'objet peut aussi être situé à côté du dispositif et il s'agit alors de « cape externe ». On ne saurait oublier de préciser que l'existence d'une cape conférant ces propriétés doit être démontrée, ce qui va dépendre du type d'équation (parabolique, hyperbolique, elliptique...) régissant la grandeur détectée. Et lorsque cette existence est prouvée d'un point de vue conceptuel, encore faut-il pouvoir approcher les propriétés de la cape avec un milieu artificiel ; ce dernier point est en général atteint avec un métamatériau, résultat d'une structuration de la matière, ou de la construction et juxtaposition de motifs élémentaires en grand nombre. Lorsque toutes ces conditions sont réunies pour réaliser l'expérience d'invisibilité (mono-fréquentielle, monodirectionnelle), on peut alors s'intéresser à son caractère large-bande (achromaticité pour l'optique) ou à sa sensibilité à l'incidence de l'onde...

Enfin il faut ajouter que les techniques de transformation d'espace ne sont pas restreintes à l'invisibilité. D'une façon bien plus large, elles autorisent une autre façon d'appréhender la conception de systèmes pour le contrôle des écoulements des flux (transfert d'énergie). On a ainsi pu voir émerger, au-delà des capes d'invisibilité/protection, des concentrateurs et des rotateurs pour la diffusion de la chaleur en thermodynamique, de la masse en biologie (avec des applications potentielles dans la pharmacie galénique grâce à un contrôle accru de la vitesse de diffusion de médicaments enrobés de capes biochimiques fortement anisotropes) ou de la lumière dans des milieux diffusants...

Les capes d'invisibilité guident les ondes autour d'une zone en créant un trou dans la métrique de l'espace, mais ce contrôle radical de leurs trajectoires reste un réel défi pour une implémentation à l'échelle du génie civil, car elle nécessite de réaliser des sols structurés avec une anisotropie artificielle extrême. Nous verrons dans le paragraphe suivant que d'autres approches innovantes issues de la physique des métamatériaux peuvent d'ores et déjà être mises en œuvre théoriquement pour la protection sismique.

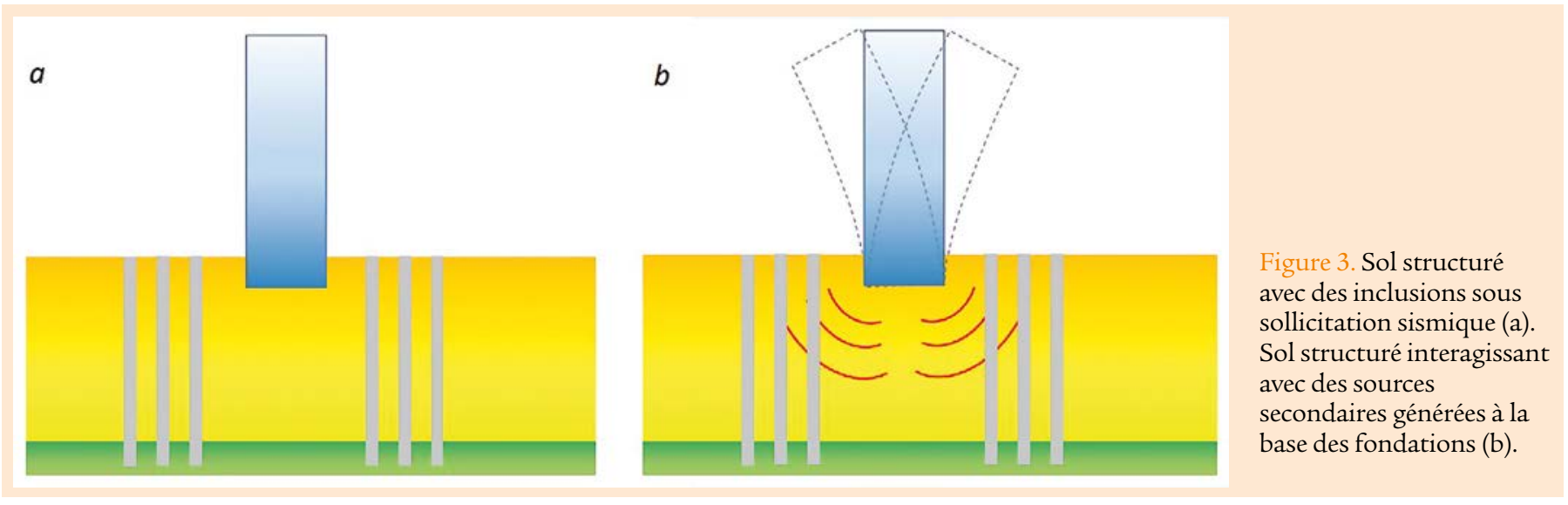




\section{Métamatériaux sismiques}

\section{Infrastructure et métamatériaux}

La notion de sols structurés est ancienne. En effet, l'Homme bâtisseur réalise depuis longtemps des sols composites pour la réalisation de fondations supportant les charges des ouvrages de surface. Depuis l'Antiquité, les constructeurs ont compris que le battage de nombreux pieux en bois dans un sol «mou » permet d'offrir une meilleure capacité portante effective [2].

Quant aux techniques modernes de fondations profondes (pieux, barrettes, parois moulées, etc.) et de renforcement de sol (inclusions rigides, inclusions souples, etc.), elles évoluent vers l'augmentation des longueurs des éléments constitutifs avec des profondeurs atteignant 50 à $70 \mathrm{~m}$. Aussi, l'industrialisation de ces procédés permet un degré croissant de densification des éléments incorporés dans le sol hôte, au point qu'il convient de s'interroger sur les nouvelles propriétés mécaniques de ce milieu « composite», sous sollicitation statique ou dynamique.

Ces milieux composites sont aussi le plus souvent structurés, c'est-à-dire qu'ils possèdent une distribution spatiale non aléatoire des inclusions, que nous définissons comme tout élément mis en place dans le sol, quelle que soit sa géométrie, ou du sol modifié (sol densifié, sol imprégné d'un coulis ou d'une résine, etc.). Cette structuration par l'action de l'Homme renvoie à la notion de « métamatériau » décrite depuis une dizaine d'années pour les ondes électromagnétiques. Ces métamatériaux sont souvent périodiques avec une périodicité $a$ très inférieure à la longueur $\lambda$ du signal les sollicitant $(<50$ $\mathrm{Hz}$, soit des longueurs d'onde de surface de quelques mètres à quelques dizaines de mètres dans un sol sédimentaire tel que dans la région grenobloise ou l'Aquila en Italie) et offrent des propriétés « exotiques » et inexistantes à l'état naturel. Il s'agit notamment d'un indice de réfraction négatif ne répondant pas à la loi de Snell-Descartes applicable à l'optique géométrique.

Le terme de métamatériau sismique a tout d'abord concerné le sol lui-même, dans son plein volume, puis s'est simultanément développé avec le concept de résonateurs spécifiquement dimensionnés, toujours présents dans le sol et le concept de résonateurs de surface. Très récemment, la notion a commencé à s'étendre à des éléments de la structure de l'ouvrage avec une prise en compte des propriétés des matériaux auxétiques² par exemple [3].

\section{De l'approche pseudo-statique à la dynamique}

Les sols composites étudiés en condition pseudo-statique font l'objet d'une attention spécifique notamment quand il s'agit d'obtenir une augmentation du module de cisaillement du sol initial pour limiter la déformation du massif sol-fondations profondes. En dimensionnement des structures, le problème dynamique est souvent converti en

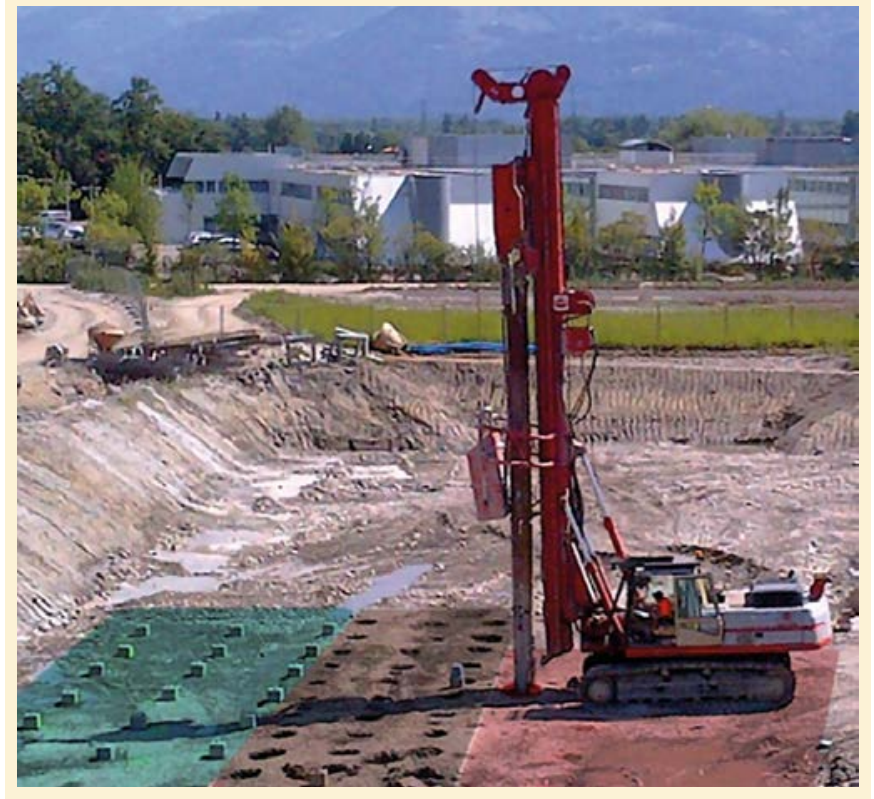

Figure 4. Métamatériau sismique en sol troué : une source vibre à $50 \mathrm{~Hz}$, et génère des ondes de surface (de Rayleigh) réfléchies par un réseau de trous de $0,3 \mathrm{~m}$ de diamètre, $5 \mathrm{~m}$ de profondeur, espacés de $1,7 \mathrm{~m}$ (distance de centre à centre) grâce à l'existence d'une bande interdite autour de $50 \mathrm{~Hz}$.

problème statique équivalent (analyse pseudo-statique), à partir d'un chargement temporel ou d'un spectre. Ainsi, l'un des intérêts principaux du développement des métamatériaux est la valorisation de l'étude fréquentielle.

Les études récentes sur les sols structurés réactivent notamment les travaux menés sur les barrières anti-vibratiles pour des sources telles que les trafics routier ou ferroviaire. Cependant, les expériences réalisées en vraie grandeur sont rares et les gammes de fréquences sont plutôt de plusieurs dizaines à centaines de hertz [2].

\section{Sols structurés et interaction sol-structure}

Les sols structurés interviennent dans le concept de l'interaction sol-structure (ISS) ou l'interaction sol-fondation-structure. Il s'agit d'une approche spécifique à la justification des ouvrages sous séisme.

Le premier effet de l'ISS à prendre en compte est le mouvement du sol, induit par le séisme, imposé à la structure et aux fondations. Selon une approche de "propagation d'onde ", l'interaction cinématique traduit l'incompatibilité entre le champ d'onde incident et les mouvements possibles de la fondation (figure 2a). En effet, la fondation est souvent plus rigide que le sol environnant et ne peut accommoder qu'une partie des déplacements induits par les ondes sismiques incidentes. Une partie de l'énergie des ondes est réfléchie, occasionnant toutefois des efforts significatifs sur la fondation, mais réduisant l'énergie transférée à la structure. Il s'agit d'un premier filtrage du signal incident. 


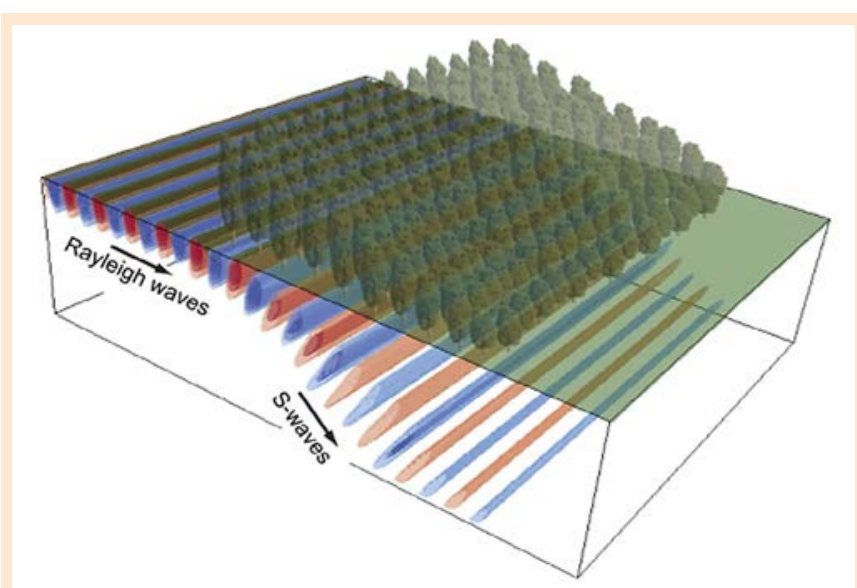

Figure 5. Infographie de métamatériau sismique (A. Colombi) en forêt d'arbres de hauteurs variables permettant la conversion d'une onde de surface (de Rayleigh) en onde de volume (de cisaillement).

Le second effet est dit « inertiel ». Pour le bâtiment cela se traduit par du balancement et du glissement. Lors d'un tremblement de terre, les ondes sismiques se propageant dans le sol mettent en mouvement les fondations des ouvrages en les sollicitant principalement horizontalement. Accéléré à sa base, chaque bâtiment est soumis à des forces d'inertie auxquelles sa structure doit résister. Pour maintenir son équilibre, l'ouvrage exerce sur le sol environnant des efforts importants : c'est ce que l'on appelle l'effet inertiel (ou couplage). Toujours selon une approche de "propagation d'ondes ", l'effet inertiel peut être décrit comme un ensemble de sources secondaires, de fréquences différentes, générées par l'ouvrage sur le sol (figure 2b).

Les sols structurés peuvent ainsi agir sur ces deux effets : modification du champ libre lié à la source sismique du tremblement de terre, et interaction avec les sources locales sous fondations (figure 3).

\section{Stratégies et applications}

Dans le cas de séismes, les ondes de pression sont couplées aux ondes de cisaillement, ce qui complique substantiellement le design d'une chape (mécanique) dans les solides : les équations de l'élasticité établies par le mathématicien français Navier ne sont malheureusement pas invariantes par transformation géométrique, et conduisent à des tenseurs à quatre indices (des tableaux de coefficients à quatre entrées). Néanmoins, il est possible d'imaginer qu'une telle chape ou structure sismique de génie civil, placée autour ou au droit des fondations d'un immeuble, réduirait sensiblement, au moins pour certaines fréquences, l'effet local d'un séisme ou encore de sources anthropiques telles que les machines vibrantes utilisées en travaux publics ou pour les équipements industriels. Un prototype très préliminaire de chape a été testé en 2012 [3] avec pour objectif de confirmer l'analogie des cristaux phononiques avec les sols structurés (cf. figure 4). Le principe de la lentille sismique est plutôt un repliement d'espace, qu'un trou dans l'espace. Le réseau de trous se comporte comme un sol avec une anisotropie élevée autour de la fréquence à $50 \mathrm{~Hz}$ de la source. Cela permet de rediriger les ondes de Rayleigh un peu à l'instar d'un miroir. Un effet miroir similaire a d'ailleurs été observé dans un réseau d'arbres sur le campus de l'université Joseph-Fourier à Grenoble [4] .

Néanmoins, pour éviter de renvoyer les ondes àl'envoyeur, il est possible de transformer les ondes de surface (appelées ondes de Rayleigh) en ondes de volume qui disparaîtront dans le sol après conversion (cf. figure 5). Le mécanisme mis en jeu avec ce qu'il convient d'appeler une méta-forêt s'appuie sur la physique des métamatériaux, chaque rangée d'arbres se comportant comme un milieu effectif aux propriétés non classiques avec, par exemple, une masse et un module de Young qui peuvent prendre des valeurs négatives pour des fréquences proches de la résonance des arbres. Si l'on conforme les rangées d'arbres sur des cercles concentriques, alors on crée un métamatériau pour les ondes sismiques avec une zone d'exclusion au centre, ce qui est l'un des objectifs du projet ANR METAFORET [5].

\section{Conclusion}

Dans cette revue des avancées récentes en sols structurés pour le contrôle des ondes de Rayleigh, nous avons mis en exergue les analogies qui existent entre les domaines a priori assez éloignés de la photonique, du génie civil et la géophysique. Des stratégies de protection antisismique ont été détaillées s'appuyant sur des concepts de miroirs réfléchissant les ondes de surface par l'effet dit de bandes interdites, et de capes sismiques, détournant les ondes à la surface un peu à l'instar des guides d'ondes pour la lumière, ou les convertissant en ondes de volume. D’autres idées émergent actuellement dans le domaine des métamatériaux sismiques, telles des structures non-réciproques, dont les pierres de touche sont la magnéto-optique et la photonique non-hermitienne où des concepts de gain compensant les pertes inhérentes dans les milieux structurés permettent de réaliser de nouvelles fonctionnalités telles que des réseaux de Bragg présentant de l'invisibilité unidirectionnelle, qui pourraient là encore apporter des solutions innovantes au génie civil, notamment sur les sources vibratiles telles que celles générées par des équipements de travaux publics, par les machines vibrantes, ou encore la circulation ferroviaire.

\section{POUR EN SAVOIR PLUS}

[1] L. Provino, L. Brilland, A. Monteville, D. Landais, O. Le Goffic, D. Tregoat, D. Mechin, Les fibres optiques microstructurées, Photoniques 65, 47 (2013)

[2] S. Anantha Ramakrishna, T.M. Grzegorczyk, Physics and Applications of Negative Refractive Index Materials, CRC Press and SPIE Press (2008).

[3] S. Brûlé, S. Enoch, S. Guenneau, Sols structurés sous sollicitation dynamique : des métamatériaux en géotechnique, Revue Française de Géotechnique 151, 1 (2017)

[4] A. Colombi, P. Roux, S. Guenneau, P. Guéguen, R.V. Craster, Forests as a natural seismic metamaterial: Rayleigh wave bandgaps induced by local resonances, Scientific Reports 6, 19238 (2016)

[5] Projet ANR METAFORET, https://metaforet.osug.fr/ 